Article

\title{
On Modified Interval-Valued Variational Control Problems with First-Order PDE Constraints
}

\author{
Savin Treanţă $\mathbb{D}$ \\ Department of Applied Mathematics, University Politehnica of Bucharest, 060042 Bucharest, \\ Romania; savin.treanta@upb.ro
}

Received: 11 February 2020; Accepted: 12 March 2020; Published: 17 March 2020

check for updates

\begin{abstract}
In this paper, a modified interval-valued variational control problem involving first-order partial differential equations (PDEs) and inequality constraints is investigated. Specifically, under some generalized convexity assumptions, we formulate and prove LU-optimality conditions for the considered interval-valued variational control problem. In order to illustrate the main results and their effectiveness, an application is provided.
\end{abstract}

Keywords: interval-valued variational control problem; invexity; pseudo-invexity; symmetry

MSC: 26B25; 65K10; 90C26; 90C30

\section{Introduction}

In recent years, saddle-point optimality criteria and the modified objective function method in optimization problems have been investigated. In this regard, we mention the works of Sposito and David [1], Smith and Vandelinde [2], Duc et al. [3], Li [4] and Santos et al. [5]. In order to solve the initial optimization problem and the corresponding duals, many researchers have been interested in obtaining new and easier methods by considering some associated optimization problems. For instance, Antczak [6], by a modification of the objective function, formulated an equivalent vector programming problem for the considered multiobjective programming problem having invex objective and constraint functions. Bhatia [7] introduced higher-order strong convexity associated with Lipschitz functions in order to derive the optimality conditions for a multiobjective optimization problem. Jayswal et al. [8], by using the second-order $\eta$-approximation method, investigated a class of vector optimization problems involving second-order invex functions. Singh et al. [9] formulated Lagrange-type dual model for a mathematical programming problem with equilibrium constraints and derived weak and strong duality results under convexity assumptions. Quite recently, Borisov and Cardone [10] studied the spectrum of a quadratic operator pencil with a small PT-symmetric periodic potential and a fixed localized potential. Under some invexity and $(\rho ; b)$-quasi-invexity assumptions of the involved functionals, Treanţă [11] established some efficiency conditions for a class of variational control problems with data uncertainty. In this paper, taking into account the applications of interval analysis in various fields and motivated and inspired by the above mentioned works, we extend the previous studies for a new class of interval-valued variational control problems with mixed constraints involving first-order partial differential equations (PDEs). Specifically, based on a class of interval-valued variational control problems recently introduced by Treanţă [11], we formulate and prove LU-optimality conditions in the considered first-order PDE-constrained modified interval-valued variational control problem. More precisely, the main novelty of this paper (compared to Treanţă [11]) is the modified objective function approach for first-order PDE-constrained interval-valued variational control problems governed by multiple integral functionals. It can be easily observed that the modified interval-valued variational control problem is simpler to study than the initial interval-valued 
variational control problem. Consequently, the present study provides important mathematical tools and ideas for further research in various fields.

The paper is structured as follows. Section 2 contains notations, definitions and the preliminary results to be used in the sequel. Section 3 includes the main results of the present paper. Concretely, a modified interval-valued variational control problem governed by first-order partial differential equations and inequality constraints is introduced. Under invexity and pseudo-invexity hypotheses, some connections between the original interval-valued variational control problem and the modified interval-valued variational control problem are established. In order to illustrate the mathematical development and its effectiveness, we present an application. Finally, Section 4 gives the conclusions of the present study.

\section{Notations and Preliminaries}

In this section, we introduce the notations, working hypotheses and the preliminary results to be used throughout the present paper. Thus, we consider:

* $\Omega \subset \mathbb{R}^{m}$ is a compact domain and $t=\left(t^{\alpha}\right), \alpha=\overline{1, m}$ is a point in $\Omega$;

* let $\mathcal{X}$ be the space of piecewise smooth state functions $x: \Omega \rightarrow \mathbb{R}^{n}$ with the norm

$$
\|x\|=\|x\|_{\infty}+\sum_{\alpha=1}^{m}\left\|x_{\alpha}\right\|_{\infty}, \quad \forall x \in \mathcal{X},
$$

where $x_{\alpha}$ denotes $\frac{\partial x}{\partial t^{\alpha}}$

* also, denote by $\mathcal{U}$ the space of piecewise continuous control functions $u: \Omega \rightarrow \mathbb{R}^{k}$ with the uniform norm $\|\cdot\|_{\infty}$;

* for $\mathcal{P}:=\Omega \times \mathbb{R}^{n} \times \mathbb{R}^{k}$, we define the following continuously differentiable functions

$$
\begin{gathered}
X=\left(X_{\alpha}^{i}\right): \mathcal{P} \rightarrow \mathbb{R}^{n m}, \quad i=\overline{1, n}, \alpha=\overline{1, m}, \\
Y=\left(Y_{\beta}\right): \mathcal{P} \rightarrow \mathbb{R}^{q}, \quad \beta=\overline{1, q} ;
\end{gathered}
$$

* $\quad d v:=d t^{1} d t^{2} \cdots d t^{m}$ represents the volume element on $\mathbb{R}^{m} \supset \Omega$;

* we assume that the continuously differentiable functions

$$
X_{\alpha}=\left(X_{\alpha}^{i}\right): \mathcal{P} \rightarrow \mathbb{R}^{n}, \quad i=\overline{1, n}, \alpha=\overline{1, m},
$$

fulfill the complete integrability conditions, that is,

$$
D_{\zeta} X_{\alpha}^{i}=D_{\alpha} X_{\zeta}^{i}, \quad \alpha, \zeta=\overline{1, m}, \alpha \neq \zeta, i=\overline{1, n},
$$

where $D_{\zeta}$ is the total derivative operator;

* for $w=\left(w_{1}, \ldots, w_{p}\right), l=\left(l_{1}, \ldots, l_{p}\right)$ in $\mathbb{R}^{p}$, the following convention will be used throughout the paper:

$$
\begin{gathered}
w=l \Leftrightarrow w_{i}=l_{i}, \quad w \leq l \Leftrightarrow w_{i} \leq l_{i}, \\
w<l \Leftrightarrow w_{i}<l_{i}, \quad w \preceq l \Leftrightarrow w \leq l, w \neq l, \quad i=\overline{1, p} .
\end{gathered}
$$

In the following, in order to formulate and prove the main results included in this paper, we present the invexity and pseudo-invexity associated with a multiple integral functional.

Consider a continuously differentiable function

$$
h: J^{1}\left(\mathbb{R}^{m}, \mathbb{R}^{n}\right) \times \mathbb{R}^{k} \rightarrow \mathbb{R}, \quad h=h\left(t, x(t), x_{\alpha}(t), u(t)\right),
$$


where $J^{1}\left(\mathbb{R}^{m}, \mathbb{R}^{n}\right)$ is the first-order jet bundle associated to $\mathbb{R}^{m}$ and $\mathbb{R}^{n}$. For $x \in \mathcal{X}$ and $u \in \mathcal{U}$, we introduce the following scalar functional

$$
H: \mathcal{X} \times \mathcal{U} \rightarrow \mathbb{R}, \quad H(x, u)=\int_{\Omega} h\left(t, x(t), x_{\alpha}(t), u(t)\right) d v .
$$

Taking into account Treanţă [12], according to Treanţă and Arana-Jiménez [13,14] and following Mititelu and Treanţă [15], we formulate the next definitions. Further, we use the notation

$$
\left(\Lambda_{x u}\right)=\left(t, x(t), u(t), x^{0}(t), u^{0}(t)\right) .
$$

Definition 1. If there exist

$$
\begin{gathered}
\eta: \Omega \times \mathbb{R}^{n} \times \mathbb{R}^{k} \times \mathbb{R}^{n} \times \mathbb{R}^{k} \rightarrow \mathbb{R}^{n}, \\
\eta=\eta\left(\Lambda_{x u}\right)=\left(\eta_{i}\left(\Lambda_{x u}\right)\right), \quad i=\overline{1, n},
\end{gathered}
$$

of $C^{1}$-class with $\eta\left(\Lambda_{x^{0} u^{0}}\right)=0, \forall t \in \Omega,\left.\eta\right|_{\partial \Omega}=0$, and

$$
\begin{gathered}
\xi: \Omega \times \mathbb{R}^{n} \times \mathbb{R}^{k} \times \mathbb{R}^{n} \times \mathbb{R}^{k} \rightarrow \mathbb{R}^{k}, \\
\xi=\xi\left(\Lambda_{x u}\right)=\left(\xi_{j}\left(\Lambda_{x u}\right)\right), \quad j=\overline{1, k},
\end{gathered}
$$

of $C^{0}$-class with $\xi\left(\Lambda_{x^{0} u^{0}}\right)=0, \forall t \in \Omega,\left.\xi\right|_{\partial \Omega}=0$, such that for every $(x, u) \in \mathcal{X} \times \mathcal{U}$ :

$$
\begin{gathered}
H(x, u)-H\left(x^{0}, u^{0}\right) \\
\geq \int_{\Omega}\left[h_{x}\left(t, x^{0}(t), x_{\alpha}^{0}(t), u^{0}(t)\right) \eta+h_{x_{\alpha}}\left(t, x^{0}(t), x_{\alpha}^{0}(t), u^{0}(t)\right) D_{\alpha} \eta\right] d v \\
+\int_{\Omega}\left[h_{u}\left(t, x^{0}(t), x_{\alpha}^{0}(t), u^{0}(t)\right) \xi\right] d v,
\end{gathered}
$$

then $H$ is said to be invex at $\left(x^{0}, u^{0}\right) \in \mathcal{X} \times \mathcal{U}$ with respect to $\eta$ and $\xi$.

Definition 2. If there exist

$$
\begin{gathered}
\eta: \Omega \times \mathbb{R}^{n} \times \mathbb{R}^{k} \times \mathbb{R}^{n} \times \mathbb{R}^{k} \rightarrow \mathbb{R}^{n}, \\
\eta=\eta\left(\Lambda_{x u}\right)=\left(\eta_{i}\left(\Lambda_{x u}\right)\right), \quad i=\overline{1, n},
\end{gathered}
$$

of $C^{1}$-class with $\eta\left(\Lambda_{x^{0} u^{0}}\right)=0, \forall t \in \Omega,\left.\eta\right|_{\partial \Omega}=0$, and

$$
\begin{gathered}
\xi: \Omega \times \mathbb{R}^{n} \times \mathbb{R}^{k} \times \mathbb{R}^{n} \times \mathbb{R}^{k} \rightarrow \mathbb{R}^{k}, \\
\xi=\xi\left(\Lambda_{x u}\right)=\left(\xi_{j}\left(\Lambda_{x u}\right)\right), \quad j=\overline{1, k},
\end{gathered}
$$

of $C^{0}$-class with $\xi\left(\Lambda_{x^{0} u^{0}}\right)=0, \forall t \in \Omega,\left.\xi\right|_{\partial \Omega}=0$, such that for every $(x, u) \in \mathcal{X} \times \mathcal{U}$ :

$$
\begin{gathered}
H(x, u)-H\left(x^{0}, u^{0}\right)<0 \\
\Rightarrow \int_{\Omega}\left[h_{x}\left(t, x^{0}(t), x_{\alpha}^{0}(t), u^{0}(t)\right) \eta+h_{x_{\alpha}}\left(t, x^{0}(t), x_{\alpha}^{0}(t), u^{0}(t)\right) D_{\alpha} \eta\right] d v \\
+\int_{\Omega}\left[h_{u}\left(t, x^{0}(t), x_{\alpha}^{0}(t), u^{0}(t)\right) \xi\right] d v<0,
\end{gathered}
$$

or, equivalently,

$$
\int_{\Omega}\left[h_{x}\left(t, x^{0}(t), x_{\alpha}^{0}(t), u^{0}(t)\right) \eta+h_{x_{\alpha}}\left(t, x^{0}(t), x_{\alpha}^{0}(t), u^{0}(t)\right) D_{\alpha} \eta\right] d v
$$




$$
+\int_{\Omega}\left[h_{u}\left(t, x^{0}(t), x_{\alpha}^{0}(t), u^{0}(t)\right) \xi\right] d v \geq 0 \Rightarrow H(x, u)-H\left(x^{0}, u^{0}\right) \geq 0,
$$

then $H$ is said to be pseudo-invex at $\left(x^{0}, u^{0}\right) \in \mathcal{X} \times \mathcal{U}$ with respect to $\eta$ and $\xi$.

Further, let $K$ be the set of all closed and bounded real intervals. Denote by $A=\left[a^{L}, a^{U}\right]$ a closed and bounded real interval, where $a^{L}$ and $a^{U}$ indicate the lower and upper bounds of $A$, respectively. Throughout this paper, the interval operations can be performed as follows:

(1) $\quad A=B \Longrightarrow a^{L}=b^{L}$ and $a^{U}=b^{U}$;

(2) if $a^{L}=a^{U}=a$ then $A=[a, a]=a$;

(3) $A+B=\left[a^{L}+b^{L}, a^{U}+b^{U}\right]$;

(5) $-A=-\left[a^{L}, a^{U}\right]=\left[-a^{U},-a^{L}\right]$;

(5) $A-B=\left[a^{L}-b^{U}, a^{U}-b^{L}\right]$;

(6) $k+A=\left[k+a^{L}, k+a^{U}\right], k \in \mathbb{R}$;

(7) $k A=\left[k a^{L}, k a^{U}\right], k \in \mathbb{R}, k \geq 0$;

(8) $k A=\left[k a^{U}, k a^{L}\right], k \in \mathbb{R}, k<0$.

Definition 3. ([11]) Let $A, B \in K$ be two closed and bounded real intervals. We write $A \preceq_{L U} B$ if and only if $a^{L} \leq b^{L}$ and $a^{U} \leq b^{U}$.

Definition 4. ([11]) Let $A, B \in K$ be two closed and bounded real intervals. We write $A \prec_{L U} B$ if and only if $A \preceq_{L U} B$ and $A \neq B$.

Definition 5. ([11]) A function $f: \mathcal{P} \rightarrow K$, defined by

$$
f \chi_{x u}(t)=\left[f^{L} \chi_{x u}(t), f^{U} \chi_{x u}(t)\right], \quad t \in \Omega,
$$

where $\chi_{x u}(t):=(t, x(t), u(t)), f^{L} \chi_{x u}(t)$ and $f^{U} \chi_{x u}(t)$ are real-valued functions and satisfy the condition $f^{L} \chi_{x u}(t) \leq f^{U} \chi_{x u}(t), t \in \Omega$, is said to be interval-valued function.

Now, we introduce the following class of interval-valued variational control problems, where the objective functional $F(x, u)=\int_{\Omega} f \chi_{x u}(t) d v,(x, u) \in \mathcal{X} \times \mathcal{U}$, is considered as interval-valued (for more details, see Treanţă [11]):

$$
\begin{gathered}
(C P) \min _{(x, u)}\left\{\int_{\Omega} f \chi_{x u}(t) d v=\left[\int_{\Omega} f^{L} \chi_{x u}(t) d v, \int_{\Omega} f^{U} \chi_{x u}(t) d v\right]\right\} \\
\text { subject to } \\
\frac{\partial x^{i}}{\partial t^{\alpha}}(t)=X_{\alpha}^{i} \chi_{x u}(t), \quad i=\overline{1, n}, \alpha=\overline{1, m}, t \in \Omega, \\
Y \chi_{x u}(t) \leq 0, \quad t \in \Omega, \\
\left.x(t)\right|_{\partial \Omega}=\varphi(t)=\text { given, }
\end{gathered}
$$

where $f: \mathcal{P} \rightarrow K$ is an interval-valued function and $f^{L}, f^{U}: \mathcal{P} \rightarrow \mathbb{R}$ are continuously differentiable real-valued functions.

Define the set $\mathcal{D}$ of all feasible solutions (domain) in $(C P)$ as

$$
\mathcal{D}:=\{(x, u) \mid x \in \mathcal{X}, u \in \mathcal{U} \text { satisfying (1), (2), (3) }\} .
$$

Definition 6. ([11]) A feasible solution $\left(x^{0}, u^{0}\right) \in \mathcal{D}$ in interval-valued variational control problem $(C P)$ is called LU-optimal solution if there exists no other feasible solution $(x, u) \in \mathcal{D}$ such that $F(x, u) \prec_{L U} F\left(x^{0}, u^{0}\right)$. 
The next result formulates necessary LU-optimality conditions for a feasible point in $(C P)$. In the following, summation over the repeated indices is assumed.

Theorem 1. (Necessary LU-optimality conditions, [11]) Under constraint qualification assumptions, if $\left(x^{0}, u^{0}\right) \in \mathcal{D}$ is an LU-optimal solution in $(C P)$, then there exists the piecewise smooth functions $\theta: \Omega \rightarrow \mathbb{R}^{2}, \theta(t)=\left(\theta^{L}(t), \theta^{U}(t)\right), \mu: \Omega \rightarrow \mathbb{R}^{q}$ and $\lambda: \Omega \rightarrow \mathbb{R}^{n m}$, with $\mu(t)=\left(\mu^{\beta}(t)\right) \in \mathbb{R}^{q}, \lambda(t)=$ $\left(\lambda_{i}^{\alpha}(t)\right) \in \mathbb{R}^{n m}$, such that:

$$
\begin{gathered}
\theta^{L}(t) \frac{\partial f^{L}}{\partial x^{i}} \chi_{x^{0} u^{0}}(t)+\theta^{U}(t) \frac{\partial f^{U}}{\partial x^{i}} \chi_{x^{0} u^{0}}(t)+\lambda_{i}^{\alpha}(t) \frac{\partial X_{\alpha}^{i}}{\partial x^{i}} \chi_{x^{0} u^{0}}(t) \\
+\mu^{\beta}(t) \frac{\partial Y_{\beta}}{\partial x^{i}} \chi_{x^{0} u^{0}}(t)+\frac{\partial \lambda_{i}^{\alpha}}{\partial t^{\alpha}}(t)=0, \quad i=\overline{1, n}, \\
\theta^{L}(t) \frac{\partial f^{L}}{\partial u^{j}} \chi_{x^{0} u^{0}}(t)+\theta^{U}(t) \frac{\partial f^{U}}{\partial u^{j}} \chi_{x^{0} u^{0}}(t)+\lambda_{i}^{\alpha}(t) \frac{\partial X_{\alpha}^{i}}{\partial u^{j}} \chi_{x^{0} u^{0}}(t) \\
+\mu^{\beta}(t) \frac{\partial Y_{\beta}}{\partial u^{j}} \chi_{x^{0} u^{0}}(t)=0, \quad j=\overline{1, k}, \\
\mu^{\beta}(t) Y_{\beta} \chi_{x^{0} u^{0}}(t)=0 \quad(\text { no summation), } \quad(\theta(t), \mu(t)) \succeq 0,
\end{gathered}
$$

for all $t \in \Omega$, except at discontinuities.

Definition 7. ([11]) The LU-optimal solution $\left(x^{0}, u^{0}\right) \in \mathcal{D}$ in $(C P)$ is said to be a normal LU-optimal solution if $\theta(t)>0$.

\section{Main Results}

This section includes the main results of the present paper. More precisely, we define a modified interval-valued variational control problem associated with $(C P)$ and, under some invexity and pseudo-invexity assumptions, we establish LU-optimality conditions for the considered interval-valued optimization problems.

For an arbitrary given feasible solution $\left(x^{0}, u^{0}\right) \in \mathcal{D}$ in $(C P)$ and for $\eta, \xi$ defined as in Definitions 1 and 2 , we introduce a modified interval-valued variational control problem associated with $(C P)$, as follows:

$$
\begin{gathered}
\left(C P_{\eta, \xi}\left(x^{0}, u^{0}\right)\right) \quad \min _{(x, u)} \int_{\Omega}\left(f_{x} \chi_{x^{0} u^{0}}(t) \eta+f_{u} \chi_{x^{0} u^{0}}(t) \xi\right) d v \\
\text { subject to } \\
\frac{\partial x^{i}}{\partial t^{\alpha}}(t)=X_{\alpha}^{i} \chi_{x u}(t), \quad i=\overline{1, n}, \alpha=\overline{1, m}, t \in \Omega, \\
Y \chi_{x u}(t) \leq 0, \quad t \in \Omega, \\
\left.x(t)\right|_{\partial \Omega}=\varphi(t)=\text { given, }
\end{gathered}
$$

where

$$
\begin{gathered}
\int_{\Omega}\left(f_{x} \chi_{x^{0} u^{0}}(t) \eta+f_{u} \chi_{x^{0} u^{0}}(t) \xi\right) d v \\
:=\left[\int_{\Omega}\left(f_{x}^{L} \chi_{x^{0} u^{0}}(t) \eta+f_{u}^{L} \chi_{x^{0} u^{0}}(t) \xi\right) d v, \int_{\Omega}\left(f_{x}^{u} \chi_{x^{0} u^{0}}(t) \eta+f_{u}^{U} \chi_{x^{0} u^{0}}(t) \xi\right) d v\right] .
\end{gathered}
$$

Remark 1. We observe that the set of all feasible solutions for the considered modified interval-valued control problem $\left(C P_{\eta, \xi}\left(x^{0}, u^{0}\right)\right)$ is $\mathcal{D}$, as well. 
Definition 8. A point $(\hat{x}, \hat{u}) \in \mathcal{D}$ is said to be an LU-optimal solution for $\left(C P_{\eta, \xi}\left(x^{0}, u^{0}\right)\right)$ if

$$
\begin{gathered}
\int_{\Omega}\left(f_{x} \chi_{x^{0} u^{0}}(t) \eta\left(\Lambda_{x u}\right)\right) d v+\int_{\Omega}\left(f_{u} \chi_{x^{0} u^{0}}(t) \xi\left(\Lambda_{x u}\right)\right) d v \\
\succeq_{L U} \int_{\Omega}\left(f_{x} \chi_{x^{0} u^{0}}(t) \eta\left(\Lambda_{\hat{x} \hat{u}}\right)\right) d v+\int_{\Omega}\left(f_{u} \chi_{x^{0} u^{0}}(t) \xi\left(\Lambda_{\hat{x} \hat{u}}\right)\right) d v,
\end{gathered}
$$

for every $(x, u) \in \mathcal{D}$.

The concept of a normal LU-optimal solution associated with the modified interval-valued control problem $\left(C P_{\eta, \xi}\left(x^{0}, u^{0}\right)\right)$ has the same meaning as in Definition 7.

Further, under some invexity assumptions, we establish the equivalence between LU-optimal solutions associated with $(C P)$ and $\left(C P_{\eta, \xi}\left(x^{0}, u^{0}\right)\right)$.

Theorem 2. Consider $\left(x^{0}, u^{0}\right) \in \mathcal{D}$ is a normal LU-optimal solution in $(C P)$ and $\int_{\Omega} \mu^{\beta}(t) Y_{\beta} \chi_{x u}(t) d v, \int_{\Omega} \lambda_{i}^{\alpha}(t)\left(X_{\alpha}^{i} \chi_{x u}(t)-\frac{\partial x^{i}}{\partial t^{\alpha}}(t)\right) d v$ are invex at $\left(x^{0}, u^{0}\right) \in \mathcal{D}$ with respect to $\eta$ and $\xi$. Then $\left(x^{0}, u^{0}\right) \in \mathcal{D}$ is an LU-optimal solution in $\left(C P_{\eta, \xi}\left(x^{0}, u^{0}\right)\right)$.

Proof. By hypothesis, the relations in Equations (4)-(6), with $\theta(t)=(1,1)$ (for instance), are satisfied for all $t \in \Omega$, except at discontinuities. By reductio ad absurdum, consider that $\left(x^{0}, u^{0}\right) \in \mathcal{D}$ is not an LU-optimal solution in $\left(C P_{\eta, \xi}\left(x^{0}, u^{0}\right)\right)$. Thus, there exists $(\bar{x}, \bar{u}) \in \mathcal{D}$ such that

$$
\begin{gathered}
\int_{\Omega}\left(f_{x} \chi_{x^{0} u^{0}}(t) \eta\left(\Lambda_{\overline{x u}}\right)\right) d v+\int_{\Omega}\left(f_{u} \chi_{x^{0} u^{0}}(t) \xi\left(\Lambda_{\overline{x u}}\right)\right) d v \\
\prec_{L U} \int_{\Omega}\left(f_{x} \chi_{x^{0} u^{0}}(t) \eta\left(\Lambda_{x^{0} u^{0}}\right)\right) d v+\int_{\Omega}\left(f_{u} \chi_{x^{0} u^{0}}(t) \xi\left(\Lambda_{x^{0} u^{0}}\right)\right) d v .
\end{gathered}
$$

Taking into account that

$$
\eta\left(\Lambda_{x^{0} u^{0}}\right)=0, \quad \xi\left(\Lambda_{x^{0} u^{0}}\right)=0, \quad \forall t \in \Omega,
$$

we get

$$
\int_{\Omega}\left(f_{x} \chi_{x^{0} u^{0}}(t) \eta\left(\Lambda_{\overline{x u}}\right)\right) d v+\int_{\Omega}\left(f_{u} \chi_{x^{0} u^{0}}(t) \xi\left(\Lambda_{\overline{x u}}\right)\right) d v \prec_{L U}[0,0]
$$

Since $\int_{\Omega} \mu^{\beta}(t) Y_{\beta} \chi_{x u}(t) d v$ is invex at $\left(x^{0}, u^{0}\right) \in \mathcal{D}$ with respect to $\eta$ and $\xi$, we have

$$
\begin{gathered}
\int_{\Omega} \mu^{\beta}(t) Y_{\beta} \chi_{\overline{x u}}(t) d v-\int_{\Omega} \mu^{\beta}(t) Y_{\beta} \chi_{x^{0} u^{0}}(t) d v \\
\geq \int_{\Omega}\left(\mu^{\beta}(t)\left(Y_{\beta}\right)_{x} \chi_{x^{0} u^{0}}(t) \eta\left(\Lambda_{\overline{x u}}\right)\right) d v+\int_{\Omega}\left(\mu^{\beta}(t)\left(Y_{\beta}\right)_{u} \chi_{x^{0} u^{0}}(t) \xi\left(\Lambda_{\overline{x u}}\right)\right) d v .
\end{gathered}
$$

Using the feasibility of $\left(x^{0}, u^{0}\right)$ and $(\bar{x}, \bar{u})$, the previous inequality becomes

$$
\begin{gathered}
\int_{\Omega}\left(\mu^{\beta}(t)\left(Y_{\beta}\right)_{x} \chi_{x^{0} u^{0}}(t) \eta\left(\Lambda_{\overline{x u}}\right)\right) d v \\
+\int_{\Omega}\left(\mu^{\beta}(t)\left(Y_{\beta}\right)_{u} \chi_{x^{0} u^{0}}(t) \xi\left(\Lambda_{\overline{x u}}\right)\right) d v \leq 0 .
\end{gathered}
$$

Also, using the feasibility of $\left(x^{0}, u^{0}\right)$ and $(\bar{x}, \bar{u})$ and, as well, the invexity of $\int_{\Omega} \lambda_{i}^{\alpha}(t)\left(X_{\alpha}^{i} \chi_{x u}(t)-\frac{\partial x^{i}}{\partial t^{\alpha}}(t)\right) d v$, we get

$$
\int_{\Omega}\left[\lambda_{i}^{\alpha}(t)\left(X_{\alpha}^{i} \chi_{x^{0} u^{0}}(t)-\frac{\partial x^{i}}{\partial t^{\alpha}}(t)\right)_{x} \eta\left(\Lambda_{\overline{x u}}\right)\right] d v
$$




$$
\begin{aligned}
& +\int_{\Omega}\left[\lambda_{i}^{\alpha}(t)\left(X_{\alpha}^{i} \chi_{x^{0} u^{0}}(t)-\frac{\partial x^{i}}{\partial t^{\alpha}}(t)\right)_{x_{\alpha}} D_{\alpha} \eta\left(\Lambda_{\overline{x u}}\right)\right] d v \\
& +\int_{\Omega}\left[\lambda_{i}^{\alpha}(t)\left(X_{\alpha}^{i} \chi_{x^{0} u^{0}}(t)-\frac{\partial x^{i}}{\partial t^{\alpha}}(t)\right)_{u} \xi\left(\Lambda_{\overline{x u}}\right)\right] d v \leq 0,
\end{aligned}
$$

or, equivalently,

$$
\begin{gathered}
\int_{\Omega}\left[\lambda_{i}^{\alpha}(t)\left(X_{\alpha}^{i}\right)_{x} \chi_{x^{0} u^{0}}(t) \eta\left(\Lambda_{\overline{x u}}\right)\right] d v-\int_{\Omega}\left[\lambda_{i}^{\alpha}(t) D_{\alpha} \eta\left(\Lambda_{\overline{x u}}\right)\right] d v \\
+\int_{\Omega}\left[\lambda_{i}^{\alpha}(t)\left(X_{\alpha}^{i}\right)_{u} \chi_{x^{0} u^{0}}(t) \xi\left(\Lambda_{\overline{x u}}\right)\right] d v \leq 0,
\end{gathered}
$$

or (using the property $\left.\eta\right|_{\partial \Omega}=0$ )

$$
\begin{gathered}
\int_{\Omega}\left[\lambda_{i}^{\alpha}(t)\left(X_{\alpha}^{i}\right)_{x} \chi_{x^{0} u^{0}}(t)+D_{\alpha} \lambda_{i}^{\alpha}(t)\right] \eta\left(\Lambda_{\overline{x u}}\right) d v \\
+\int_{\Omega}\left[\lambda_{i}^{\alpha}(t)\left(X_{\alpha}^{i}\right)_{u} \chi_{x^{0} u^{0}}(t) \xi\left(\Lambda_{\overline{x u}}\right)\right] d v \leq 0 .
\end{gathered}
$$

Combining Equations (7)-(9), we obtain

$$
\begin{gathered}
\int_{\Omega}\left[\sum_{r=L, U} f_{x}^{r} \chi_{x^{0} u^{0}}(t)+\lambda_{i}^{\alpha}(t)\left(X_{\alpha}^{i}\right)_{x} \chi_{x^{0} u^{0}}(t)\right] \eta\left(\Lambda_{\overline{x u}}\right) d v \\
+\int_{\Omega}\left[\mu^{\beta}(t)\left(Y_{\beta}\right)_{x} \chi_{x^{0} u^{0}}(t)+D_{\alpha} \lambda_{i}^{\alpha}(t)\right] \eta\left(\Lambda_{\overline{x u}}\right) d v \\
+\int_{\Omega}\left[\sum_{r=L, U} f_{u}^{r} \chi_{x^{0} u^{0}}(t)+\lambda_{i}^{\alpha}(t)\left(X_{\alpha}^{i}\right)_{u} \chi_{x^{0} u^{0}}(t)\right] \xi\left(\Lambda_{\overline{x u}}\right) d v \\
\quad+\int_{\Omega}\left[\mu^{\beta}(t)\left(Y_{\beta}\right)_{u} \chi_{x^{0} u^{0}}(t)\right] \xi\left(\Lambda_{\overline{x u}}\right) d v<0 .
\end{gathered}
$$

Taking into account Equations (4) and (5) (with $\theta(t)=(1,1)$ ), we obtain a contradiction. Consequently, $\left(x^{0}, u^{0}\right) \in \mathcal{D}$ is an LU-optimal solution in $\left(C P_{\eta, \xi}\left(x^{0}, u^{0}\right)\right)$ and the proof is complete.

Illustrative application. In order to illustrate the effectiveness of the aforementioned result (see Theorem 2), consider the following bi-dimensional control problem (in short BCP):

$$
\begin{gathered}
(B C P) \quad \min _{(x, u)}\left[\int_{\Omega_{0,3}}(u(t)-4)^{2} d t^{1} d t^{2}, \int_{\Omega_{0,3}} u^{2}(t) d t^{1} d t^{2}\right] \\
\text { subject to } \\
\frac{\partial x}{\partial t^{1}}(t)=3-u(t), \quad \frac{\partial x}{\partial t^{2}}(t)=3-u(t), \quad t \in \Omega_{0,3} \\
81-x^{2}(t) \leq 0, \quad t \in \Omega_{0,3} \\
x(0)=x(0,0)=6, \quad x(3)=x(3,3)=8
\end{gathered}
$$

where $t=\left(t^{1}, t^{2}\right)$ and $\Omega_{t_{0}, t_{1}}=\Omega_{0,3}$ is a square fixed by the diagonally opposite points $t_{0}=\left(t_{0}^{1}, t_{0}^{2}\right)=$ $(0,0)$ and $t_{1}=\left(t_{1}^{1}, t_{1}^{2}\right)=(3,3)$ in $\mathbb{R}^{2}$.

The symmetry of the variables $t^{1}$ and $t^{2}$, generated by (10), plays a crucial role in our investigations, specifically, in obtaining the LU-optimal solution associated with $(B C P)$. Moreover, we consider interest only for affine state functions and $\theta=\left(\theta^{L}, \theta^{U}\right)$ is a constant function (see Theorem 1). 
In the aforementioned application, the following mathematical tools are used:

$$
\begin{gathered}
f: \Omega_{0,3} \times \mathbb{R} \times \mathbb{R} \rightarrow K, \\
X_{\alpha}: \Omega_{0,3} \times \mathbb{R} \times \mathbb{R} \rightarrow \mathbb{R}, \quad \alpha=1,2, \\
Y: \Omega_{0,3} \times \mathbb{R} \times \mathbb{R} \rightarrow \mathbb{R}, \\
f \chi_{x u}(t)=\left[f^{L} \chi_{x u}(t), f^{U} \chi_{x u}(t)\right]=\left[(u(t)-4)^{2}, u^{2}(t)\right], \\
X_{1} \chi_{x u}(t)=X_{2} \chi_{x u}(t)=3-u(t), \quad Y \chi_{x u}(t)=81-x^{2}(t),
\end{gathered}
$$

continuously differentiable functions. The closeness condition associated to $X_{\alpha}, \alpha=1,2$, implies $\frac{\partial u}{\partial t^{1}}(t)=\frac{\partial u}{\partial t^{2}}(t)$, and the condition $f^{L} \chi_{x u}(t) \leq f^{U} \chi_{x u}(t), t \in \Omega_{0,3}$, involves $u(t) \geq 2, t \in \Omega_{0,3}$.

Consider the feasible point in $(B C P)$

$$
x^{0}(t)=\frac{1}{3}\left(t^{1}+t^{2}\right)+6, \quad u^{0}(t)=\frac{8}{3}, \quad t \in \Omega_{0,3}
$$

and let $\eta, \xi: \Omega_{0,3} \times(\mathbb{R} \times \mathbb{R})^{2} \rightarrow \mathbb{R}$ be defined as

$$
\begin{aligned}
& \eta\left(\Lambda_{x u}\right)=\left\{\begin{aligned}
x(t)-x^{0}(t), & t \in \operatorname{Int}\left(\Omega_{0,3}\right) \\
0, & t \in \partial \Omega_{0,3},
\end{aligned}\right. \\
& \xi\left(\Lambda_{x u}\right)=\left\{\begin{aligned}
u(t)-u^{0}(t), & t \in \operatorname{Int}\left(\Omega_{0,3}\right) \\
0, & t \in \partial \Omega_{0,3} .
\end{aligned}\right.
\end{aligned}
$$

The modified interval-valued variational control problem associated with $(B C P)$ is formulated as

$$
\left(B C P_{\eta, \xi}\left(x^{0}, u^{0}\right)\right) \quad \min _{(x, u)}\left[\int_{\Omega_{0,3}}\left(\frac{64}{9}-\frac{8}{3} u(t)\right) d t^{1} d t^{2}, \int_{\Omega_{0,3}}\left(\frac{16}{3} u(t)-\frac{128}{9}\right) d t^{1} d t^{2}\right]
$$

subject to (10), (11), (12).

We can notice that $\left(B C P_{\eta, \xi}\left(x^{0}, u^{0}\right)\right)$ has a simplified form in comparison to $(B C P)$. Also, the feasible point $\left(x^{0}, u^{0}\right)$, defined in (13), is an LU-optimal solution for $(B C P)$. According to the relations $(4)-(6)$, with $\theta(t)=(1,1)$, it follows that $\lambda(t)=\left(c_{1}, c_{2}\right)$, where $c_{1}, c_{2}$ are real constants with $c_{1}+c_{2}=\frac{8}{3}$ and $\mu(t)=0, \forall t \in \Omega_{0,3}$. Further, it is easy to check the invexity of $\int_{\Omega} \mu^{\beta}(t) Y_{\beta} \chi_{x u}(t) d v, \int_{\Omega} \lambda_{i}^{\alpha}(t)\left(X_{\alpha}^{i} \chi_{x u}(t)-\frac{\partial x^{i}}{\partial t^{\alpha}}(t)\right) d v$ at $\left(x^{0}, u^{0}\right)$ with respect to $\eta$ and $\xi$ formulated in Equations (14) and (15). Consequently, the feasible point $\left(x^{0}, u^{0}\right)$, defined in (13), is an LU-optimal solution in $\left(B C P_{\eta, \xi}\left(x^{0}, u^{0}\right)\right)$.

The following result provides LU-optimality conditions for the reverse situation presented in the previous theorem.

Theorem 3. Consider $\left(x^{0}, u^{0}\right) \in \mathcal{D}$ is an LU-optimal solution in $\left(C P_{\eta, \xi}\left(x^{0}, u^{0}\right)\right)$ and the functionals $\int_{\Omega} f^{r} \chi_{x u}(t) d v, r=L, U$, are pseudo-invex at $\left(x^{0}, u^{0}\right) \in \mathcal{D}$ with respect to $\eta$ and $\xi$. Then $\left(x^{0}, u^{0}\right) \in \mathcal{D}$ is an LU-optimal solution in (CP).

Proof. Consider, by reductio ad absurdum, that $\left(x^{0}, u^{0}\right) \in \mathcal{D}$ is not an LU-optimal solution in $(C P)$. Therefore, there exists $(\bar{x}, \bar{u}) \in \mathcal{D}$ satisfying

$$
\int_{\Omega} f \chi_{\overline{x u}}(t) d v \prec L U \int_{\Omega} f \chi_{x^{0} u^{0}}(t) d v .
$$


Since the functionals $\int_{\Omega} f^{r} \chi_{x u}(t) d v, r=L, U$, are pseudo-invex at $\left(x^{0}, u^{0}\right) \in \mathcal{D}$ with respect to $\eta$ and $\xi$, the previous inequality implies

$$
\int_{\Omega}\left(f_{x} \chi_{x^{0} u^{0}}(t) \eta\left(\Lambda_{\overline{x u}}\right)\right) d v+\int_{\Omega}\left(f_{u} \chi_{x^{0} u^{0}}(t) \xi\left(\Lambda_{\overline{x u}}\right)\right) d v \prec_{L u}[0,0] .
$$

By using the property $\eta\left(\Lambda_{x^{0} u^{0}}\right)=\xi\left(\Lambda_{x^{0} u^{0}}\right)=0$, we can write the above inequality as follows

$$
\begin{gathered}
\int_{\Omega}\left(f_{x} \chi_{x^{0} u^{0}}(t) \eta\left(\Lambda_{\overline{x u}}\right)\right) d v+\int_{\Omega}\left(f_{u} \chi_{x^{0} u^{0}}(t) \xi\left(\Lambda_{\overline{x u}}\right)\right) d v \\
\prec_{L U} \int_{\Omega}\left(f_{x} \chi_{x^{0} u^{0}}(t) \eta\left(\Lambda_{x^{0} u^{0}}\right)\right) d v+\int_{\Omega}\left(f_{u} \chi_{x^{0} u^{0}}(t) \xi\left(\Lambda_{x^{0} u^{0}}\right)\right) d v,
\end{gathered}
$$

which contradicts the optimality of $\left(x^{0}, u^{0}\right) \in \mathcal{D}$ in $\left(C P_{\eta, \xi}\left(x^{0}, u^{0}\right)\right)$. In consequence, $\left(x^{0}, u^{0}\right) \in \mathcal{D}$ is an LU-optimal solution in $(C P)$ and the proof is complete.

\section{Conclusions}

Because of the high complexity of the environment, often are inaccurate and unreliable initial data. For example, in the modeling of many processes in industry and economy in order to make decisions, it is not always possible to have complete information about the parameters and variables involved. Therefore, an adequate uncertainty framework is necessary to formulate the model and new methods have to be adapted or developed to provide optimal or efficient solutions in a certain sense. In order to tackle the uncertainty in an optimization problem, the interval-valued optimization represents a growing branch of applied mathematics.

In this paper, we have defined a modified interval-valued variational control problem which is easier to solve than the initial control problem. Also, for this new interval-valued variational control problem, we have formulated and proved conditions of LU-optimality. An example of interval-valued variational control problem has been presented to illustrate the results established in the paper.

Funding: The APC was funded by University Politehnica of Bucharest, "PubArt" program.

Conflicts of Interest: The author declares no conflict of interest.

\section{References}

1. Sposito, V.A.; David, H.T. Saddle-point optimality criteria of nonlinear programming problems over cones without differentiability. SIAM J. Appl. Math. 1971, 20, 698-702. [CrossRef]

2. Smith, R.H., Jr.; Vandelinde, V.D. A saddle-point optimality criterion for nonconvex programming in normed spaces. SIAM J. Appl. Math. 1972, 23, 203-213. [CrossRef]

3. Duc, D.M.; Hoang, N.D.; Nguyen, L.H. Lagrange multipliers theorem and saddle point optimality criteria in mathematical programming. J. Math. Anal. Appl. 2006, 323, 441-455. [CrossRef]

4. Li, X. Saddle-point condition for fractional programming. In Proceedings of the 2012 Eighth International Conference on Computational Intelligence and Security, Guangzhou, China, 17-18 November 2012; pp. 82-85.

5. Santos, L.B.d.; Rojas-Medar, M.A.; de Oliveira, V.A. Saddle point and second order optimality in nondifferentiable nonlinear abstract multiobjective optimization. TEMA (São Carlos) 2012, 13, $179-191$. [CrossRef]

6. Antczak, T. A new approach to multiobjective programming with a modified objective function. J. Glob. Optim. 2003, 27, 485-495. [CrossRef]

7. Bhatia, G. Optimality and mixed saddle point criteria in multiobjective optimization. J. Math. Anal. Appl. 2008, 342, 135-145. [CrossRef]

8. Jayswal, A.; Jha, S.; Choudhury, S. Saddle point criteria for second order $\eta$-approximated vector optimization problems. Kybernetika 2016, 52, 359-378. [CrossRef] 
9. Singh, Y.; Pandey, Y.; Mishra, S.K. Saddle point optimality criteria for mathematical programming problems with equilibrium constraints. Oper. Res. Lett. 2017, 45, 254-258. [CrossRef]

10. Borisov, D.; Cardone, G. Spectra of operator pencils with small PT-symmetric periodic perturbation. ESAIM Control. Optim. Calc. Var. 2020, 26. [CrossRef]

11. Treanţă S. Efficiency in uncertain variational control problems. Neural Comput. Applic. 2020, in press.

12. Treanţă, S. On a modified optimal control problem with first-order PDE constraints and the associated saddle-point optimality criterion. Eur. J. Control 2020, 51, 1-9. [CrossRef]

13. Treanţă, S.; Arana-Jiménez, M. KT-pseudo-invex multidimensional control problem. Optim. Control Appl. Meth. 2018, 39, 1291-1300. [CrossRef]

14. Treanţă, S.; Arana-Jiménez, M. On generalized KT-pseudo-invex control problems involving multiple integral functionals. Eur. J. Control 2018, 43, 39-45. [CrossRef]

15. Mititelu, Ş.; Treanţă S. Efficiency conditions in vector control problems governed by multiple integrals. J. Appl. Math. Comput. 2018, 57, 647-665. [CrossRef]

(C) 2020 by the author. Licensee MDPI, Basel, Switzerland. This article is an open access article distributed under the terms and conditions of the Creative Commons Attribution (CC BY) license (http:/ / creativecommons.org/licenses/by/4.0/). 